\title{
Telehealth for chronic disease management
}

In this cluster randomised controlled trial, Cartwright et al ${ }^{1}$ evaluated the outcomes of telehealth on patient reported outcomes in a primary care population with chronic disease. Telehealth is the use of remote data exchange between patients at home and health professionals to assist management of long-term conditions. It is heralded as a solution to our growing elderly population with its significant chronic disease burden. The Department of Health has commissioned Whole Systems Demonstrator (WSD) to analyse the efficacy and cost-effectiveness of telehealth. The WSD study consists of a series of nested analyses in a chronic disease population. This paper looks specifically at patient reported anxiety, depression and health-related quality of life.

A total of 1573 patients at 154 practices across three sites were recruited and had to have at least one chronic disease (chronic obstructive pulmonary disease, heart failure or diabetes), a broadband internet connection, English literacy and sufficient cognitive ability to complete the questionnaire unaided. Telehealth systems varied and included blood pressure, blood glucose, weight and pulse oximetry measurement as appropriate for the patient and at individualised intervals, symptom data entry and educational support. Monitoring centres were staffed by nursing and support staff with a variety of possible interventions such as physical or telephone consultation.

The main endpoints were health-related quality of life, depression and anxiety at 12 months measured via home interview with a trained researcher using widely accepted inventories. Neither the intention to treat analysis nor the per protocol analysis found any significant difference between the treatment and control arms in terms of health-related quality of life, anxiety or depression.

This is the largest and most comprehensive investigation of telehealth to date, and the authors suggest that previous trials which have found a beneficial or inconclusive effect on patient reported outcomes were frequently small or methodologically weak. The authors postulate that patients may prefer face-to-face consultations and dislike continual self-monitoring. Further analyses on cost-effectiveness, admission prevention and disease outcomes from the WSD trial are awaited, but this constitutes strong evidence that psychological outcomes are not improved by telehealth.

Aarash Saleh

Correspondence to Dr Aarash Saleh, Whipps Cross University Hospital, Whipps Cross Road, London E11 1NR, UK; aarash.saleh@nhs.net

Competing interests None.

Provenance and peer review Not commissioned; internally peer reviewed.

To cite Saleh A. Thorax 2014;69:334.

Published Online First 11 July 2013

Thorax 2014;69:334. doi:10.1136/thoraxjn-2013-204082

\section{REFERENCE}

1 Cartwright M, Hirani SP, Rixon L, et al. Whole Systems Demonstrator Evaluation Team. Effect of telehealth on quality of life and psychological outcomes over 12 months (Whole Systems Demonstrator telehealth questionnaire study): nested study of patient reported outcomes in a pragmatic, cluster randomised controlled trial. BMJ 2013;346:f653. 